\title{
Evaluation of Idiopathic Choroidal Neovascularization with Indocyanine Green Angiography in Patients Undergoing Bevacizumab Therapy
}

\author{
Ryan B. Rush ${ }^{1,2,3}$ and Sloan W. Rush ${ }^{2,3}$ \\ ${ }^{1}$ Southwest Retina Specialists, 7411 Wallace Boulevard, Amarillo, TX 79106, USA \\ ${ }^{2}$ Panhandle Eye Group, 7400 Fleming Avenue, Amarillo, TX 79106, USA \\ ${ }^{3}$ Texas Tech University Health Science Center, 1400 S. Coulter, Amarillo, TX 79106, USA \\ Correspondence should be addressed to Ryan B. Rush; ryanbradfordrush21@hotmail.com
}

Received 15 March 2015; Revised 17 May 2015; Accepted 24 May 2015

Academic Editor: Enrico Peiretti

Copyright ( 2015 R. B. Rush and S. W. Rush. This is an open access article distributed under the Creative Commons Attribution License, which permits unrestricted use, distribution, and reproduction in any medium, provided the original work is properly cited.

Purpose. To examine the clinical implications of change in choroidal neovascularization (CNV) size on indocyanine green (ICG) angiography in subjects with idiopathic CNV undergoing bevacizumab therapy. Methods. The charts of subjects with an idiopathic CNV treated by a modified PRN regimen with intravitreal bevacizumab over a 12-month period were retrospectively reviewed. Results. There were 34 subjects included in the analysis. Baseline CNV sizes of less than $1.0 \mathrm{~mm}^{2}$ on ICG angiography correlated with complete CNV resolution $(P=0.0404)$, fewer injections delivered $(P=0.0002)$, and better Snellen visual acuity $(P=0.0098)$ at 12 months. Subjects that experienced a 33\% or more reduction in CNV size on ICG angiography at 2 months had complete CNV resolution $(P=0.0047)$ and fewer injections $(P<0.0001)$ at 12 months compared to subjects that did not experience a $33 \%$ or more reduction in CNV size on ICG angiography at 2 months. Conclusions. Smaller baseline CNV size on ICG angiography resulted in better visual acuity and fewer injections at 12 months, and a reduction of $33 \%$ or more in CNV size after 2 months may predict a better clinical course in subjects with idiopathic CNV undergoing bevacizumab therapy.

\section{Introduction}

Idiopathic choroidal neovascularization (CNV) is a diagnosis of exclusion occurring in subjects younger than 50 years without any observable ocular or systemic disease associations $[1,2]$. Although permanent visual loss can occur without treatment, the natural history and visual outcomes of idiopathic CNV are generally more favorable than CNV attributable to age-related macular degeneration (AMD) [3]. Several studies have reported favorable visual and anatomic outcomes in subjects with idiopathic CNV undergoing antivascular endothelial growth factor (VEGF) therapy [4-10].

Baseline CNV size has been described as an important prognostic indicator of response to anti-VEGF therapy in neovascular AMD and pathologic myopia [11, 12]. Recently, indocyanine green (ICG) angiography with the Heidelberg Spectralis (Heidelberg Engineering, Heidelberg, Germany) system has been reported to be an accurate and reproducible method for obtaining measurements of CNV secondary to AMD and pathologic myopia $[13,14]$. It remains unknown if change in CNV size in response to anti-VEGF therapy in patients with idiopathic $\mathrm{CNV}$ can predict the clinical course for these patients. In this study, the authors investigate the clinical correlations and implications that change in CNV size on ICG angiography has on treatment-naïve subjects with idiopathic CNV undergoing bevacizumab therapy during a 12-month follow-up interval.

\section{Methods}

The SRS institutional review board (IORG0007600/ IRB00009122) approved this retrospective chart review of subjects with idiopathic CNV treated with bevacizumab 
from January 2009 to December 2014 at a single privatepractice institution. All research components adhered to the tenets of the Declaration of Helsinki and were conducted in agreement with human research standards and regulations.

The inclusion criteria for the study were as follows: (1) age was $<50$ years; (2) a diagnosis of treatment-naïve subfoveal $\mathrm{CNV}$ was demonstrated by clinical examination, fluorescein angiography (FA), optical coherence tomography (OCT), and ICG angiography; (3) baseline Snellen best-corrected visual acuity (BCVA) was between 20/25 and 20/200; (4) baseline central macular thickness on OCT was >320 microns; (5) a 12month follow-up period was fully documented with interval BCVA, examination details, OCT, FA, and ICG angiography; ICG angiography must have been performed at baseline, 2 months ( \pm one month), 6 months ( \pm 2 months), and 12 months ( \pm 2 months); (6) a pro re nata (PRN) regimen was adhered to as described below; and (7) there were no identifiable primary ocular or systemic diseases associated with the CNV.

The exclusion criteria for the study were as follows: (1) photodynamic therapy, macular photocoagulation, or intravitreal injections other than bevacizumab to the study eye were given during the study period, (2) media opacity was significantly affecting BCVA or hindering retinal examination or imaging acquisition, (3) intraocular surgery for any indication was performed during the study period or within 3 months prior to subject enrollment, (4) visually significant retinal diseases other than idiopathic $\mathrm{CNV}$, active uveitis, or uncontrolled glaucoma were present at enrollment or developed during the study period, and (5) a previous vitrectomy was performed at any time to the study eye (before or during the study period).

Baseline examinations included BCVA, an anterior and posterior segment slit-lamp examination, OCT, FA, and ICG angiography. Subjects received the initial bevacizumab injection during the baseline evaluation, and further bevacizumab injections were determined according to the following PRN schedule: consecutive monthly bevacizumab injections were given until the macula was dry (without intraretinal or subretinal fluid) on OCT and without macular hemorrhage on fundus examination. Once these measures were achieved, bevacizumab therapy would be deferred and the patient would return for follow-up four weeks later. If retinal fluid recurred on OCT, macular hemorrhage developed, or BCVA dropped by 2 or more lines after injection deferral, treatment would then be reinitiated. If subjects demonstrated resolution of CNV on ICG angiography or were without retinal edema on OCT for three consecutive months, follow-up was extended out to $8( \pm 4)$ weeks' interval.

The Heidelberg Spectralis system was used for all OCT, FA, and ICG angiography procedures. Intraretinal and/or subretinal fluid and central macular thickness were determined by OCT. Central macular thickness was obtained from the center subfield of the macular thickness map. The technique for determining CNV size on ICG angiography with the Heidelberg Spectralis surface area-measuring software has been previously described [14]. Briefly, an early to midframe (within the first three minutes) ICG angiography test image was selected for analysis. The visible CNV
TABLE 1: The baseline means and distributions of the study population.

\begin{tabular}{lc}
\hline Variable & Outcome \\
\hline Age (years) & $38.7( \pm 10.0)$ \\
\hline Gender & $\begin{array}{c}\text { Female: } 19(55.8 \%) \\
\text { Male: } 15(44.1 \%)\end{array}$ \\
\hline Best-corrected visual acuity (logMAR) & $0.52( \pm 0.20)$ \\
\hline $\begin{array}{l}\text { Central macular thickness on optical } \\
\text { coherence tomography (microns) }\end{array}$ & $355.8( \pm 38.3)$ \\
\hline $\begin{array}{l}\text { Choroidal neovascularization surface } \\
\text { on indocyanine green angiography } \\
\left(\text { mm }{ }^{2}\right)\end{array}$ & $1.82( \pm 1.69)$ \\
\hline $\begin{array}{l}\text { Choroidal neovascularization surface } \\
\text { on fluorescein angiography }\left(\mathrm{mm}^{2}\right)\end{array}$ & $2.36( \pm 1.91)$ \\
\hline
\end{tabular}

margins were manually encircled by two masked observers independently using the "Inlay" function, followed by surface area computation with the software. If $\mathrm{CNV}$ measurements did not agree within $10 \%$ of the lesion size between masked observers, the patient was then excluded from the analysis. $\mathrm{CNV}$ was considered resolved when the CNV could no longer be visualized on ICG angiography by either masked observer.

2.1. Main Outcomes and Statistical Analysis. The study's main outcomes were CNV surface area change on ICG angiography at 2 months, 6 months, and 12 months. The secondary outcomes were the correlation of change in CNV surface area on ICG angiography with change in BCVA, change in central macular thickness on OCT, and the number of injections delivered over the 12-month study interval. Response to treatment was considered significant when the CNV surface area change on ICG angiography was $33 \%$ or more from the selected follow-up interval. The JMP software package (JMP Version 10, SAS Institute, Cary, NC, USA) was used for statistical calculations. Snellen BCVA was changed into a logarithm of the minimal angle of resolution (logMAR). Nominal variables were compared by Chi-Square and Likelihood Ratios, and numerical means were compared by oneway analysis of variance (ANOVA). A probability of $<0.05$ was deemed statistically significant.

\section{Results}

There were 37 subjects that met the study's inclusion/exclusion criteria. Of these 37 subjects, 34 were included in the data analysis after CNV measurements were assessed for interobserver agreement, thereby resulting in 91.8\% (34/37) concordance between masked observers within $10 \%$ of the lesion size on ICG angiography. As a comparative check on ICG angiography, interobserver agreement within $10 \%$ of the lesion size on FA was $67.5 \%$ (25/37). The means and distributions of the study population's baseline characteristics are summarized in Table 1. Baseline CNV size on FA was overestimated by $22.7 \%\left(2.36 \mathrm{~mm}^{2}\right.$ versus $\left.1.82 \mathrm{~mm}^{2}\right)$ when compared to ICG angiography. 


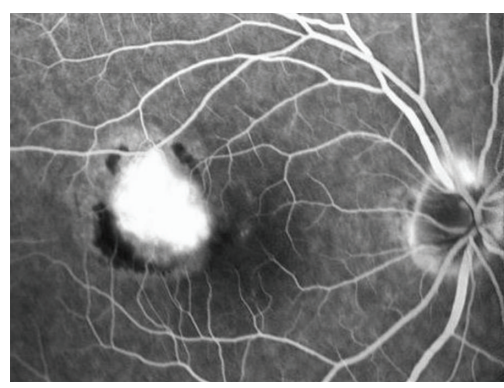

(a)

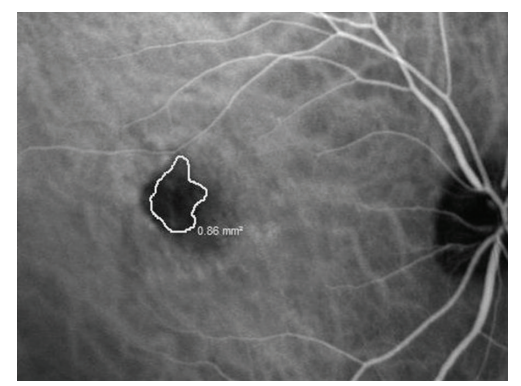

(b)

FIGURE 1: The baseline fluorescein angiography (a) and indocyanine green angiography (b) images of a 37-year-old subject with subfoveal idiopathic choroidal neovascularization. The choroidal neovascularization is encircled in white with the corresponding surface area calculation on the indocyanine green angiography image. The Snellen visual acuity was 20/80.

There was a mean of $3.7( \pm 1.9)$ injections delivered over the 12-month study interval. The mean BCVA at 12 months was $0.34( \pm 0.24) \operatorname{logMAR}(20 / 44$ Snellen), and the mean central macular thickness on OCT at 12 months was 278.3 $( \pm 36.1)$ microns. The changes in BCVA and central macular thickness on OCT during the study interval were significantly improved $(P=0.0072$ and $P<0.0001$, resp.). A total of $26.4 \%$ of subjects (9/34) improved 3 or more lines of BCVA at 12 months. There were no subjects that experienced a loss of 3 or more lines of BCVA during the study interval.

The mean CNV surface areas on ICG angiography were $1.11( \pm 1.58), 0.33( \pm 0.58)$, and $0.15( \pm 0.42) \mathrm{mm}^{2}$ at 2 months, 6 months, and 12 months, respectively. The change in CNV size on ICG angiography from baseline was significantly improved at 6 months and 12 months $(P<0.0001$ for both), but not at 2 months $(P=0.1207)$. A total of $35.2 \%(12 / 34)$ of subjects had complete CNV resolution on ICG angiography at 2 months, $70.5 \%(24 / 34)$ at 6 months, and $85.2 \%(29 / 34)$ at 12 months. A total of $11.7 \%(4 / 34)$ of subjects only required one injection to achieve complete CNV resolution on ICG angiography during the 12-month study interval, and a total of $26.4 \%$ (9/34) of subjects required two or fewer injections to achieve complete CNV resolution on ICG angiography during the 12-month study interval. The recurrence rate of $\mathrm{CNV}$ on ICG angiography after 3 monthly bevacizumab injections was $32.3 \%$ (11/34) during the study interval. A total of $61.7 \%$ (21/34) of subjects had a $33 \%$ or more CNV size reduction on ICG angiography at 2 months; subjects that experienced a $33 \%$ or more reduction in CNV size on ICG angiography at 2 months had more complete CNV resolution at 12 months $(P=0.0047)$ and fewer injections delivered during the study interval $(P<0.0001)$, but not better visual acuity $(P=$ $0.0721)$ or a lower central macular thickness on OCT $(P=$ $0.7656)$ at the end of the 12 -month study interval compared to those subjects without a 33\% or more reduction at 2 months. Using linear regression analysis, change in CNV size on ICG angiography correlated poorly with change in BCVA $(P=$ $\left.0.6836 ; R^{2}=0.006\right)$ but correlated well with change in central macular thickness on OCT $\left(P=0.0452 ; R^{2}=0.14\right)$ during the study interval. See Figures 1-4 for a case example.

Baseline CNV sizes of less than $1.0 \mathrm{~mm}^{2}$ on ICG angiography significantly correlated with complete CNV resolution

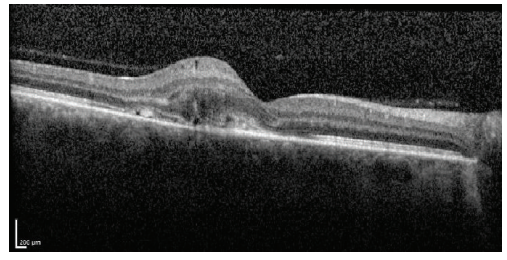

FIGURE 2: The baseline optical coherence tomography image of the same subject from Figure 1. Notice the presence of retinal fluid and choroidal neovascularization in the subretinal space.

at 12 months $(P=0.0404)$, fewer injections delivered during the study interval $(P=0.0002)$, and better visual acuity $(P=0.0098)$, but not lower central macular thickness on OCT $(P=0.2899)$ at the end of the 12-month study interval. Overall, $100 \%$ of subjects that had a baseline CNV size on ICG angiography of less than $1.0 \mathrm{~mm}^{2}$ had complete CNV resolution at 12 months.

\section{Discussion}

The baseline characteristics and overall outcomes in our study were broadly on par to other idiopathic CNV studies evaluating response to anti-VEGF therapy [4-10]. Similar to the mean 3.3 injections delivered over the first 12 months by Kang and Koh [4], our study subjects received 3.7 injections during the 12-month study interval. Our study is one of the largest case series of idiopathic CNV subjects undergoing anti-VEGF therapy, and the favorable visual and anatomic outcomes achieved in our study lend further confidence in the effectiveness of intravitreal bevacizumab for the management of idiopathic CNV.

This is the first study to investigate change in CNV size on ICG angiography in treatment-naïve patients with idiopathic CNV undergoing anti-VEGF therapy; previous studies either did not report CNV size [5-8] or measured CNV size with FA, reporting only the greatest linear dimension of the CNV leakage [4]. The authors of this study assessed CNV surface area on ICG angiography with the Heidelberg Spectralis software, believing this would provide a more objective and reproducible method for determining 


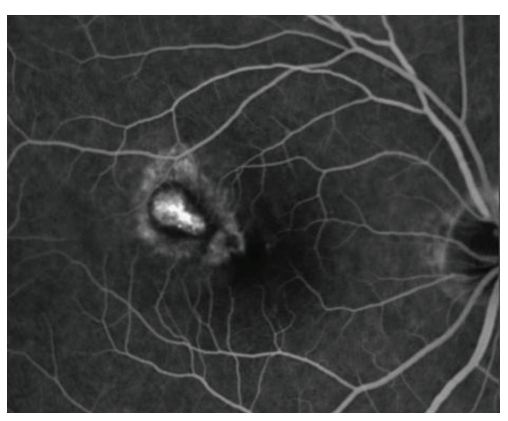

(a)

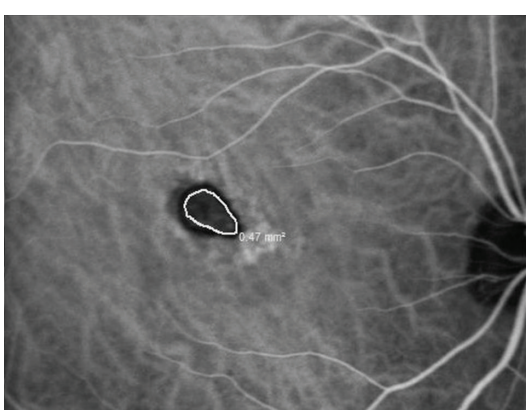

(b)

FIGURE 3: The fluorescein angiography (a) and indocyanine green angiography (b) images of the same subject presented in Figures 1 and 2 two months after two consecutive monthly bevacizumab injections were given. The choroidal neovascularization has decreased $45.3 \%$ $((0.86-0.47) / 0.86)$ from its baseline value on indocyanine green angiography. The Snellen visual acuity improved to $20 / 30$.

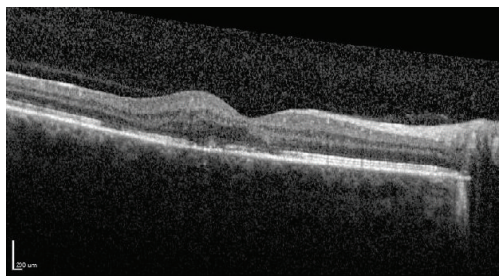

FIgURE 4: The optical coherence tomography image of the same subject presented in Figures 1-3 two months after two consecutive monthly bevacizumab injections were given. Notice the resolution of most of the retinal fluid and the choroidal neovascularization diminishment within the subretinal space.

CNV size compared to a FA classification system based on determination of hyperfluorescence and dye leakage. Indeed, when FA was used to measure CNV surface area during our study as a comparative check on ICG angiography, masked interobserver agreement was notably less and CNV size was evidently overestimated. ICG angiography has been reported to improve the detectability of CNV by $55-61 \%$ compared to FA in neovascular AMD patients, especially when occult CNV was present [15-19]. The authors believe that the measurement discrepancy between ICG angiography and FA can be best explained by noting that ICG angiography allows a direct measurement of the actual CNV lesion, whereas FA only allows an indirect measurement of the secondary effects of $\mathrm{CNV}$ such as retinal leaking and staining.

A reduction in CNV size on ICG angiography of 33\% or more at 2-month follow-up predicted a favorable clinical course with fewer injections needed and more CNV resolution in the study population by the end of the 12month study interval. Change in $\mathrm{CNV}$ size during the study interval correlated well with change in central macular thickness and total injection number, and subjects with smaller baseline CNV surface areas were more likely to have favorable visual outcomes with fewer injections; this highlights the importance of early detection and treatment while the CNV size remains small in order to get the best final outcomes. Change in CNV size did not correlate well with change in visual acuity, and this is most likely because patients with larger baseline CNV and increased baseline central macular thickness stand to gain the most in net anatomic improvement with treatment, while having the worst potential for visual recovery on account of their disease being more advanced prior to treatment.

The mean baseline CNV size in our study population with idiopathic CNV was more analogous to that reported in myopic CNV rather than neovascular AMD [11-14]. Baseline CNV surface area measured on ICG angiography has ranged from 0.93 to $2.18 \mathrm{~mm}^{2}$ in myopic subjects [20-22] and from 1.9 to $7.1 \mathrm{~mm}^{2}$ in neovascular AMD subjects [14, 23]. Change in CNV size in our study patients in response to anti-VEGF therapy was also more comparable to what has been reported in myopic CNV subjects undergoing anti-VEGF therapy [13, 20-22]. Similar to Yang et al. [13], greater baseline CNV size in our study subjects correlated with the need for a higher number of bevacizumab injections during the study interval. The recurrence rate of CNV on ICG angiography after 3 monthly bevacizumab injections in our study was similar to the rates reported by Yang et al. [13] (23.3\%) and Lai et al. [20] (22.7\%) in eyes with myopic CNV after 3 monthly anti-VEGF injections. However, complete CNV resolution after just 1-2 monthly anti-VEGF injections was lower in our study compared to the resolution rate of $54.7 \%$ reported by Hayashi et al. [21] in subjects with myopic CNV. Nevertheless, complete CNV resolution on ICG angiography in subjects with neovascular AMD has been reported to be only $7.3 \%$ at 12-month follow-up [14]. Therefore according to baseline $\mathrm{CNV}$ size and change in CNV size in response to anti-VEGF therapy, idiopathic CNV appears to behave clinically more like myopic CNV rather than neovascular AMD.

Weaknesses of this study include the use of logMAR visual acuity, the retrospective collection of data, and the observer-dependent CNV surface area determination on ICG angiography. In conclusion, our study demonstrates that smaller baseline CNV surface area on ICG angiography results in better visual acuity and fewer injections at 12 months in subjects with idiopathic CNV and that a reduction of $33 \%$ or more in CNV size after 2-month follow-up predicts 
a better clinical course with fewer injections at 12 months. Future prospective studies are needed to further validate these findings.

\section{Conflict of Interests}

The authors declare that there is no conflict of interests regarding the publication of this paper.

\section{Authors' Contribution}

Sloan W. Rush and Ryan B. Rush were responsible for design and conduct of the study; collection, management, analysis, and interpretation of the data; and preparation, review, or approval of the paper.

\section{References}

[1] A. C. Ho, L. A. Yannuzzi, K. Pisicano, J. DeRosa, and R. P. Murphy, "The natural history of idiopathic subfoveal choroidal neovascularization," Ophthalmology, vol. 102, no. 5, pp. 782-789, 1995.

[2] P. Lempert, "Idiopathic subfoveal choroidal neovascularization," Ophthalmology, vol. 102, no. 10, pp. 1411-1412, 1995.

[3] Macular Photocoagulation Study Group, "Krypton laser photocoagulation for idiopathic neovascular lesions. Results of a randomized clinical trial," Archives of Ophthalmology, vol. 108, no. 6, pp. 832-837, 1990.

[4] H. M. Kang and H. J. Koh, "Intravitreal anti-vascular endothelial growth factor therapy versus photodynamic therapy for idiopathic choroidal neovascularization," American Journal of Ophthalmology, vol. 155, no. 4, pp. 713-719, 2013.

[5] W.-M. Chan, T. Y. Y. Lai, D. T. L. Liu, and D. S. C. Lam, "Intravitreal bevacizumab (Avastin) for choroidal neovascularization secondary to central serous chorioretinopathy, secondary to punctate inner choroidopathy, or of idiopathic origin," American Journal of Ophthalmology, vol. 143, no. 6, pp. 977-983, 2007.

[6] F. Gomi, K. Nishida, Y. Oshima et al., "Intravitreal bevacizumab for idiopathic choroidal neovascularization after previous injection with posterior subtenon triamcinolone," American Journal of Ophthalmology, vol. 143, no. 3, pp. 507-510, 2007.

[7] S. Mandal, S. Garg, P. Venkatesh, C. Mithal, R. Vohra, and A. Mehrotra, "Intravitreal bevacizumab for subfoveal idiopathic choroidal neovascularization," Archives of Ophthalmology, vol. 125, no. 11, pp. 1487-1492, 2007.

[8] M. Inoue, K. Kadonosono, Y. Watanabe et al., "Results of 1-year follow-up examinations after intravitreal bevacizumab administration for idiopathic choroidal neovascularization," Retina, vol. 30, no. 5, pp. 733-738, 2010.

[9] H.-J. Qi, X.-X. Li, and Y. Tao, "Outcome of intravitreal bevacizumab for idiopathic choroidal neovascularization in a Chinese population," Canadian Journal of Ophthalmology, vol. 45, no. 4, pp. 381-385, 2010.

[10] H. Zhang, Z.-L. Liu, P. Sun, and F. Gu, "Intravitreal bevacizumab for treatment of subfoveal idiopathic choroidal neovascularization: results of a 1-year prospective trial," American Journal of Ophthalmology, vol. 153, no. 2, pp. 300.e1-306.el, 2012.
[11] R. P. Finger, S. S. Wickremasinghe, P. N. Baird, and R. H. Guymer, "Predictors of anti-VEGF treatment response in neovascular age-related macular degeneration," Survey of Ophthalmology, vol. 59, no. 1, pp. 1-18, 2014.

[12] M. Gharbiya, F. Cruciani, F. Parisi, G. Cuozzo, S. Altimari, and S. Abdolrahimzadeh, "Long-term results of intravitreal bevacizumab for choroidal neovascularisation in pathological myopia," British Journal of Ophthalmology, vol. 96, no. 8, pp. 1068-1072, 2012.

[13] H. S. Yang, J.-G. Kim, J. T. Kim, and S. G. Joe, "Prognostic factors of eyes with naïve subfoveal myopic choroidal neovascularization after intravitreal bevacizumab," American Journal of Ophthalmology, vol. 156, no. 6, pp. 1201-1210, 2013.

[14] R. B. Rush, S. W. Rush, A. V. Aragon II, and J. E. Ysasaga, "Evaluation of choroidal neovascularization with indocyanine green angiography in neovascular age-related macular degeneration subjects undergoing intravitreal bevacizumab therapy," The American Journal of Ophthalmology, vol. 158, pp. 337-344, 2014.

[15] F. Abedi, S. Wickremasinghe, A. F. M. Islam, K. M. Inglis, and R. H. Guymer, "Anti-VEGF treatment in neovascular agerelated macular degeneration: A treat-and-extend protocol over 2 years," Retina, vol. 34, pp. 1531-1538, 2014.

[16] R. C. Watzke, M. L. Klein, C. J. Hiner, B. K. S. Chan, and D. F. Kraemer, "A comparison of stereoscopic fluorescein angiography with indocyanine green videoangiography in age-related macular degeneration," Ophthalmology, vol. 107, no. 8, pp. 16011606, 2000.

[17] U. Schneider, H. Kuck, W. Inhoffen, and I. Kreissig, "Indocyanine green angiographically well-defined choroidal neovascularization: angiographic patterns obtained using the scanning laser ophthalmoscope," German Journal of Ophthalmology, vol. 4, no. 2, pp. 67-74, 1995.

[18] S. Wolf, H. Knabben, G. Krombach, A. Schaaf, U. Solbach, and M. Reim, "Indocyanine-green angiography in patients with occult choroidal neovascularization," German Journal of Ophthalmology, vol. 5, no. 5, pp. 251-256, 1996.

[19] D. R. Guyer, L. A. Yannuzzi, J. S. Slakter et al., "Classification of choroidal neovascularization by digital indocyanine green videoangiography," Ophthalmology, vol. 103, no. 12, pp. 20542060, 1996.

[20] T. Y. Y. Lai, F. O. J. Luk, G. K. Y. Lee, and D. S. C. Lam, "Longterm outcome of intravitreal anti-vascular endothelial growth factor therapy with bevacizumab or ranibizumab as primary treatment for subfoveal myopic choroidal neovascularization," Eye, vol. 26, no. 7, pp. 1004-1011, 2012.

[21] K. Hayashi, N. Shimada, M. Moriyama, W. Hayashi, T. Tokoro, and K. Ohno-Matsui, "Two-year outcomes of intravitreal bevacizumab for choroidal neovascularization in Japanese patients with pathologic myopia," Retina, vol. 32, no. 4, pp. 687-695, 2012.

[22] M. Miyake, K. Yamashiro, Y. Akagi-Kurashige et al., "Vascular endothelial growth factor gene and the response to antivascular endothelial growth factor treatment for choroidal neovascularization in high myopia," Ophthalmology, vol. 121, no. 1, pp. 225-233, 2014.

[23] D. R. Guyer, L. A. Yannuzzi, J. S. Slakter et al., "Classification of choroidal neovascularization by digital indocyanine green video angiography," Ophthalmology, vol. 103, no. 12, pp. 20542060, 1996. 


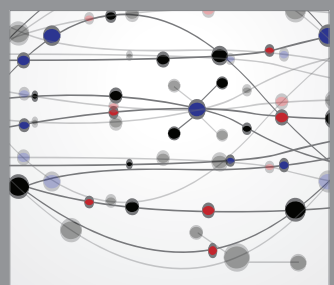

The Scientific World Journal
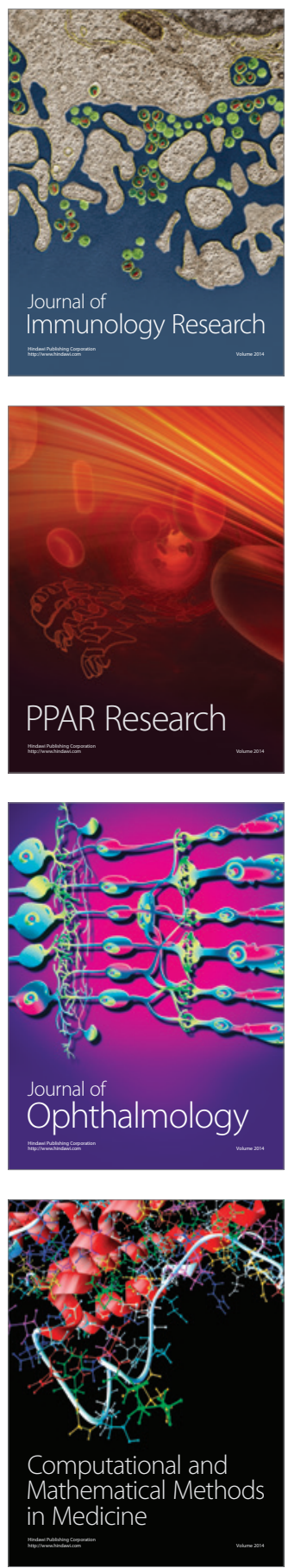

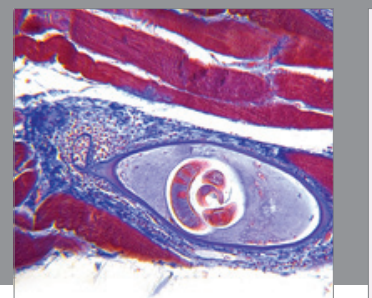

Gastroenterology

Research and Practice
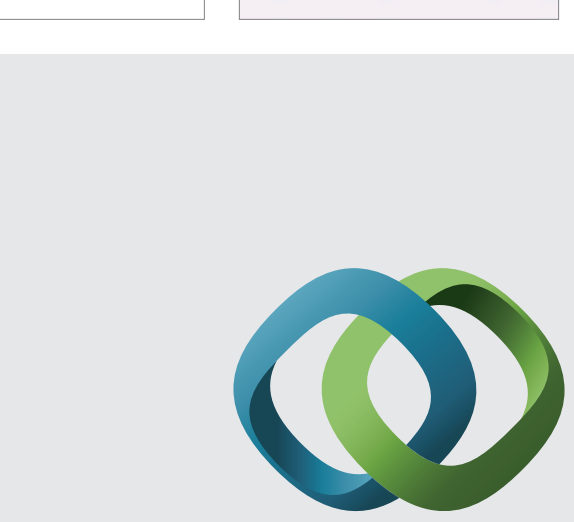

\section{Hindawi}

Submit your manuscripts at

http://www.hindawi.com
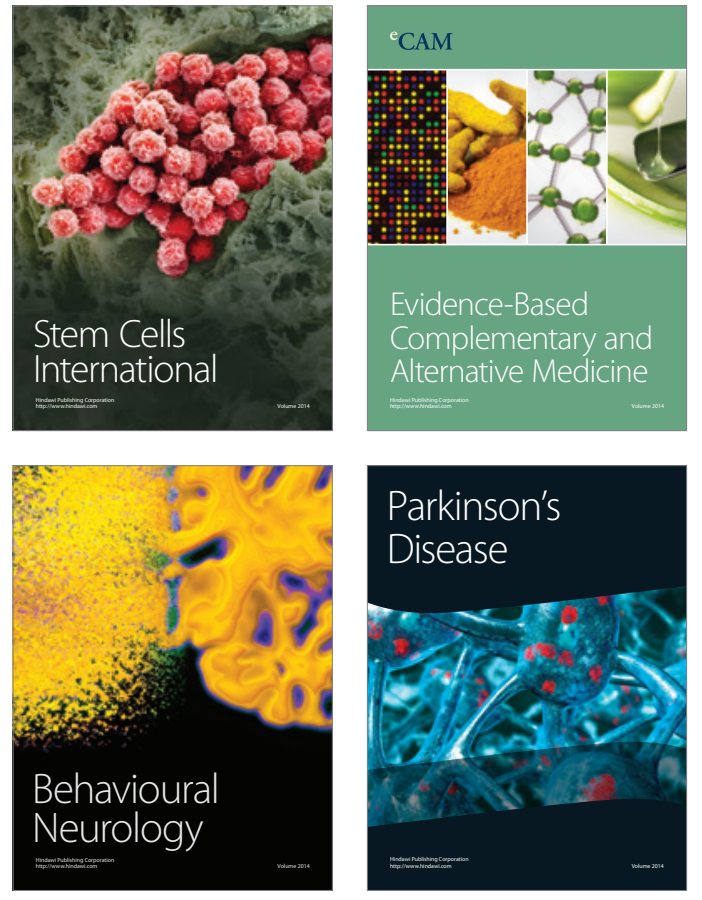
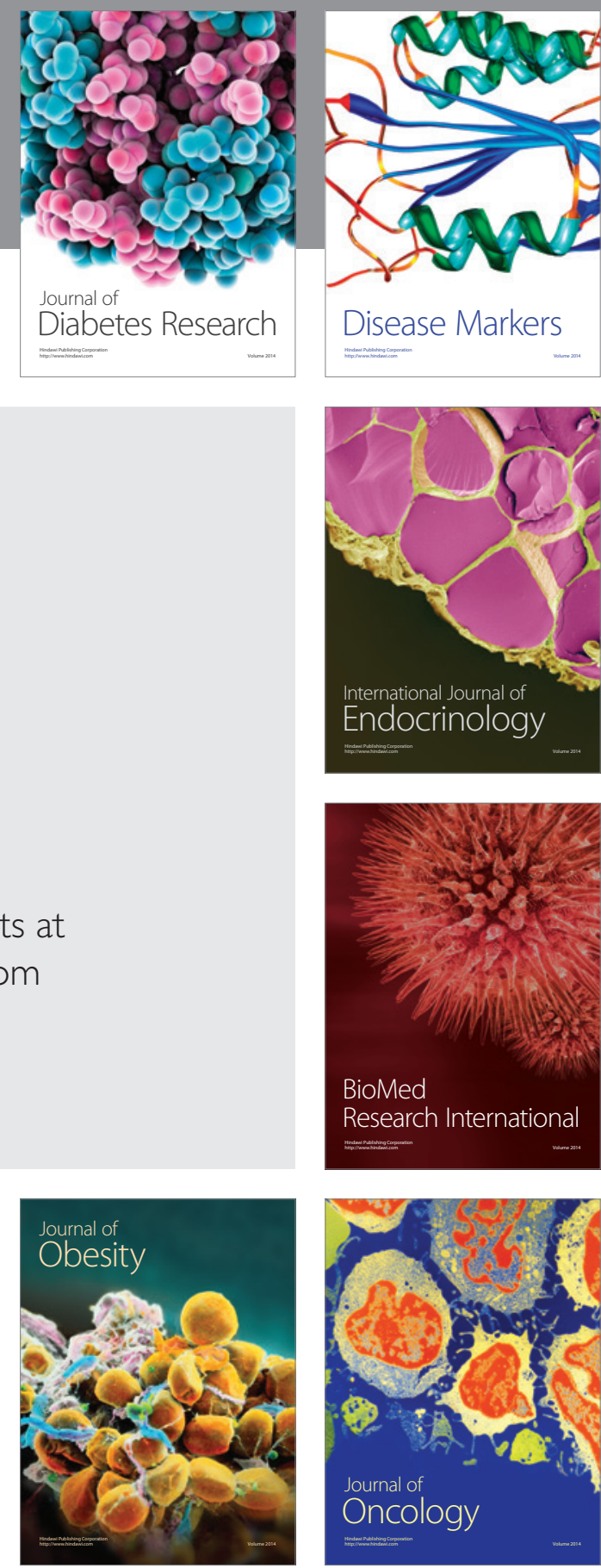

Disease Markers
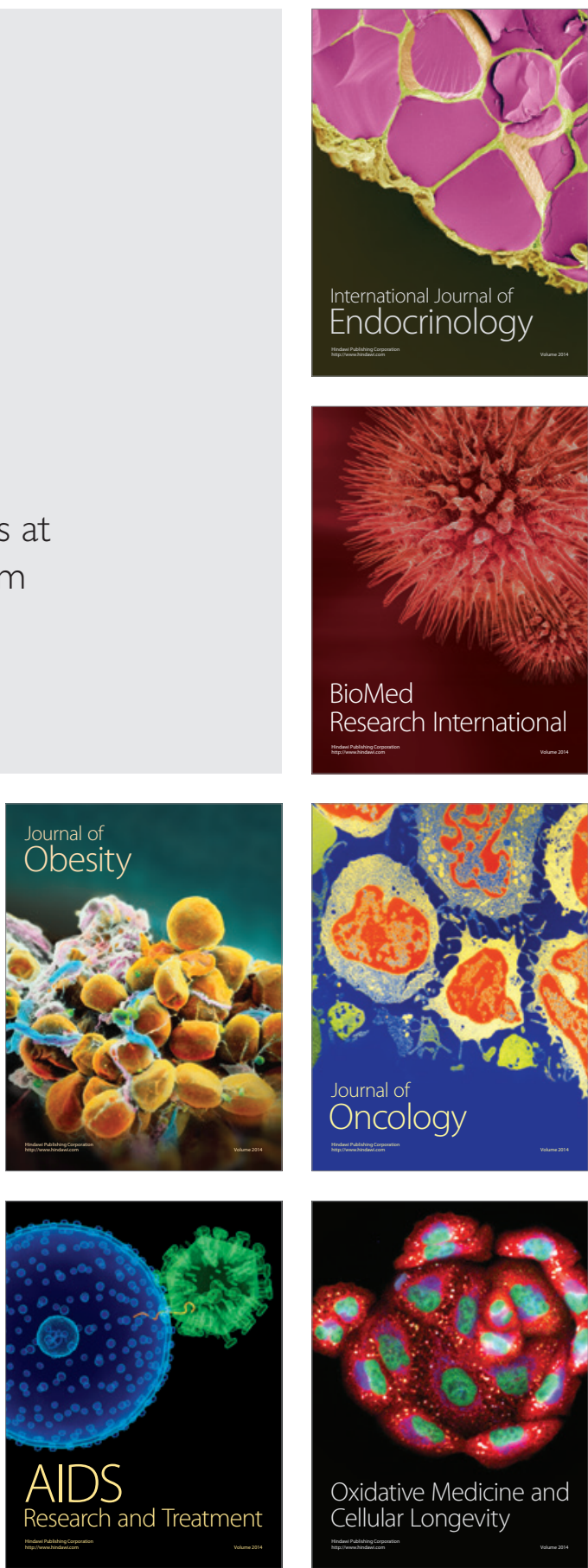